disease, including smoking, hypertension, obesity, a sedentary lifestyle and hyperlipidaemia. There is plenty of room for improvement in monitoring the physical health of patients on antipsychotic treatment.

Who should be primarily responsible for such monitoring? The American Diabetes Association and others developed a measurement consensus statement that recommended the monitoring of weight, waist measurement, blood pressure, fasting glucose and lipids (American Diabetes Association et al, 2004). Does the responsibility fall mainly to our primary care colleagues or do we need to take a more active role? Do we need better facilities for monitoring in our in-patient and community clinics with involvement of our nursing and dietician colleagues?

Action needs to be taken when any results or measurements are abnormal. Results are often communicated to general practitioners by letter and can easily be overlooked. I wonder whether we need to start initiating treatment such as statins ourselves. This would require ongoing education in primary care medicine. Other possible measures include interventions to promote general healthy living and lifestyle changes, and weight management clinics.

AMERICAN DIABETES ASSOCIATION et al (2004) Consensus development conference on antipsychotic drugs and obesity and diabetes. Diabetes Care, 27. 596-601.

Khalid Mohd. Sani Senior House Officer in Adult Psychiatry, Knowsley Assertive Outreach Team, Prysmian Building, Hall Lane, Prescot L34 5WU, email: khalidsani@hotmail.com

Atypical antipsychotic medication is used for children and adolescents, not only for childhood schizophrenia but also for adolescents with aggressive conduct disorders (Findling et al, 2000) and young people who have autistic-spectrum disorders and severe aggression (RUPP Autism Network, 2002). Unlike the adult population with enduring mental illness, research into the management of these childhood disorders is in its infancy and medication is almost always used off licence. However, child and adolescent psychiatrists in the UK have become more concerned about the metabolic side-effects of atypical antipsychotic drugs in children than about involuntary movement disorders. A consensus view is emerging that children and adolescents treated with atypical antipsychotic medication should have baseline and regular blood glucose monitoring, but there are no standards to guide and audit practice. To date it is unclear how far this practice is followed by child and adolesent psychiatrists across the country.
Most prescribing of atypical antipsychotic medication to children and adolescents will occur in out-patient rather than in-patient settings. We anticipate that the same difficulties described by Tarrant (Psychiatric Bulletin, August 2006, 30, 286-288) in the management of adult out-patients will also apply in child and adolescent psychiatry. In addition, the attitudes of practitioners, parents and children to venepuncture will influence the uptake of these tests. Some children with severe behaviour disorders cannot tolerate venepuncture. In our practice, we believe that recommending blood tests before and during treatment with atypical antipsychotic drugs emphasises the gravity of the decision to use these drugs in children

With increased concern about the level of obesity and type 2 diabetes in young people in general (Dietz, 2001), psychiatrists and general practitioners need to work closely and cooperatively to decrease the risk of iatrogenic metabolic disease in children and adolescents.

DIETZ,W. H. (2001) The obesity epidemic in young children. BMJ, 322, 313-314

FINDLING, R. L., MCNAMARA, N. K., BRANICKY, I. A. A., et al (2000) A double-blind study of risperidone in the treatment of conduct disorder. Journal of the American Academy of Child and Adolescent Psychiatry, 39, 504-516.

RUPP AUTISM NETWORK (2002) Risperidone in children with autism and serious behaviour problems. New England Journal of Medicine, 347, 314-321.

*Walid Sorour Associate Specialist in Child and Adolescent Psychiatry, Child and Adolescent Menta Health Service, Moore House, 10-11 LindumTerrace, Lincoln LN2 5RT, email: walid.sorour@lpt.nhs.uk, AnneThompson Consultant in Child and Adolescent Psychiatry, Lincoln

It is well known that psychotropic drugs can have important effects on the cardiovascular system which include changes in blood pressure and effects on the QTC interval (Pacher \& Kecskemeti, 2004). The Royal College of Psychiatrists (2006) recommends periodic monitoring by electrocardiography (ECG) during high-dose antipsychotic therapy.

We conducted a postal survey of consultant psychiatrists in the north-west of England to assess the facilities available in the psychiatric clinic for checking blood pressure and the ease of arranging ECG. We also asked whether psychiatrists were confident in interpreting ECG in order to clarify any training requirements. Out of 260 consultants, 132 returned the questionnaire (response rate $50.7 \%$ ). A majority (59\%) felt that it was difficult to arrange ECG in the clinic and worryingly an even higher proportion (61\%) lacked facilities to check blood pressure. Only a small minority $(13 \%)$ felt confident about identifying QT prolongation on ECG. Most respondents (82\%) felt that doctors working in psychiatry should have regular training in interpreting ECG.

These results demonstrate a lack of facilities in mental health clinics for cardiovascular monitoring and the need for regular training of psychiatrists in the care of the physical health of their patients.

PACHER, P. \& KECSKEMETI,V. (2004) Cardiovascular side effects of new antidepressants and antipsychotics: new drugs, old concerns? Current Pharmaceutical Design, 10, 2463-2475.

ROYAL COLLEGE OF PSYCHIATRISTS (2006) Consensus Statement on High-DoseAntipsychotic Medication (Council Report CR138). London: Royal College of Psychiatrists.

*Faouzi Alam Specialist Registrar in General Adult Psychiatry, Manchester Mental Health and Social Care Trust, MRI, Oxford Road, Manchester M13 9WL, email: docftalam@aol.com, Raghupathy Paranthaman Specialist Registrar in General Adult and Old Age Psychiatry, Manchester Mental Health and Social CareTrust, Patrick Mbaya Consultant Psychiatrist, Wythenshawe Hospital, Manchester

\section{Drug-related movement disorders: training needs}

We agree that 'psychiatrists need more structured clinical training in assessing and managing [movement] disorders to provide the best clinical care' (Kuruvilla et al, Psychiatric Bulletin, August 2006, 30, 300-303). However, we were surprised that almost all respondents thought that the training should be undertaken in the first year of the MRCPsych course. As senior psychiatric trainees who have recently attended a 'bedside' teaching session on movement disorders, we would argue that there is a need for refresher courses at a later stage of training. Effective continuing professiona development should include a regular revision of clinical skills, which cannot be achieved through reading alone. Furthermore, we feel this skill is best learnt in a small group setting with direct patient contact rather than in the more didactic MRCPsych setting. In a small group the learner is able to ask questions more freely.

Kuruvilla et al raise wider issues concerning the competency of psychiatrists in physical examination. Given the increased awareness of biological mechanisms in aetiology, particularly in old age psychiatry and liaison psychiatry, it is becoming increasingly important for psychiatrists to be competent not just in the assessment of movement disorders but all aspects of neurological examination. These are difficult skills to master and should be taught by competent teachers, often working in medical 
specialties at the clinical interface of psychiatry. Only by identifying our clinical weaknesses and then actively seeking teachers to address our learning needs will we be able ultimately to provide the best clinical care.

*AndrewTeodorczuk Academic Registrar, Wolfson Research Centre, Institute for Ageing and Health, Newcastle General Hospital, Newcastle-upon-Tyne NE4 6BE, email: andrew.teodorczuk@ncl.ac.uk, John Paul Taylor Academic Registrar, Urs P. Mosimann Clinical Senior Lecturer/Honorary Consultant in Old Age Psychiatry, Institute for Ageing and Health Wolfson Research Centre Newcastle General Hospital, Newcastle-upon-Tyne

\section{Admission is not a failure of community care}

Dr Mackirdy gives an interesting account of the pressures leading to change within adult psychiatric services (Psychiatric Bulletin, August 2006, 30, 283-285). As a member of a community mental health team I also have noted with concern what she describes as 'healthy competition between the sector teams to have the fewest in-patients', which may not be so healthy if it prevents a clinically indicated admission from taking place. I would also be unhappy with the view of in-patient admission as a 'failure of community care'. As Dr Mackirdy herself has observed, the provision of home treatment as an alternative to hospital admission has been one of the best developments of the past decade. Making an assumption that all admissions are failures of home treatment would suggest an impressive evidence base which is simply not yet available (Joy et al, 2005).

There will always be occasions when admission to a place of safety is required irrespective of the excellence of the available home treatment team (Department of Health, 2002). To view an appropriate clinical decision or indeed patient preference for admission as a failure of that service would remove any real sense of an alternative or choice for those availing of our services.

DEPARTMENT OF HEALTH (2002) Community Mental HealthTeams - Mental Health Policy Implementation Guide. London: Department of Health.

JOY, C. B., ADAMS, C. E. \& RICE, K. (2005) Crisis intervention for people with severe mental illness.
Cochrane Library, issue 3. Chichester: Wiley InterScience.

Rachel Cullivan Consultant Psychiatrist, St Davnet's Hospital, Reaskey, Monaghan, Ireland, email: rachel.cullivan@maile.hse.ie

\section{Decrease in health service use and cost following group treatment of patients with personality disorders}

It is well known that patients with personality disorders are one of the groups with the highest use of mental health services and hence one of the most expensive to treat. In an out-patient group treatment centre for personality disorders at Royal Perth Hospital, Australia, we examined service use before and after treatment. This was a retrospective analysis of 153 patients (60\% female, mean age 34 years). Treatment was for 6 months and patients attended two eclectic oriented psychotherapy groups each day for 5 days per week. Inclusion criteria were presence of one or more personality disorders. Exclusion criteria were psychosis and antisocial personality disorder.

Hospital database records were examined for the 12 months before and after treatment, and data for psychiatry in patient bed-days, out-patient visits and presentations at the emergency department were retrieved. Costs included all aspects of service delivery; for example, staff wages and building costs.

Cost was reduced from Aus $\$ 1.3$ million (Aus $\$ 8561$ per patient) in the year before treatment to Aus $\$ 556789$ (Aus $\$ 3639$ per patient) in the year after treatment. This was an offset of Aus $\$ 753073$ (Aus $\$ 4922$ per patient). These changes were significant; for example, a 65\% reduction in in-patient and bed-days, from 19 days in the year before treatment to 7 in the year after $(t(152)=2.52$ $P=0.01$ ).

Our results are similar to those of others (Dolan et al, 1996; Chiesa \& Fonagy, 2002), and suggest that mental health services should fund group psychotherapy for personality disorders as this treatment may result in cost-offsets.

CHIESA, M. \& FONAGY, P. (2002) From the therapeutic community to the community: a preliminary evaluation of a psychosocial outpatient service for severe personality disorders. Therapeutic Communities, 23, 247-258.

DOLAN B. M., WARREN, F. M., MENZIES, D., et al (1996) Cost-offset following specialist treatment of severe personality disorders. Psychiatric Bulletin, 20 $413-417$.

Sarah J. Egan Lecturer, School of Psychology, Curtin University of Technology, Perth, Australia, email: S.Egan@exchange.curtin.edu.au, Paula R. Nathan Director, Centre for Clinical Interventions, Perth, Australia

\section{Smoking and substance misuse in pregnant women with mental illness}

Shah \& Howard (Psychiatric Bulletin, August 2006, 30, 294-297) investigated smoking and substance misuse in pregnant women with mental illness. We are concerned about the interpretation of their data and the lack of adequate accounting for confounding factors.

Our recent review highlighted that the timing and level of alcohol consumption in pregnancy was important to outcome (Mukherjee et al, 2006) but this was not taken into account by Shah \& Howard. It would have been better to divide the alcohol consumption group into no alcohol, previous alcohol and ongoing alcohol consumption in order to exclude it as a confounding effect. Since $61 \%$ of the population has been shown to drink during pregnancy, and there is a large underestimate of consumption, it is a risk factor that must be adequately excluded.

This publication potentially challenges data emerging from international literature. Here the small numbers in some of the subgroups, combined with the failure to control adequately for the important risk factor that is alcohol, means that an otherwise important piece of research will have to be interpreted with caution.

MUKHERJEE, R. A. S. TURK, J. \& HOLLINS, S. (2006) Fetal alcohol spectrum disorder: an overview. Journal of the Royal Society of Medicine, 99, 298-302.

${ }^{*}$ Raja A. S. Mukherjee Consultant Psychiatrist, Surrey Border Partnership Trust and Honorary Lecturer, St George's, Univeristy of London, CranmerTerrace, London SW17 ORE, email: rmukherj@sgul.ac.uk, JeremyTurk Professor of Academic Child and Adolescent Mental Health, Division of Clinical Developmental Sciences, St George's, University of London 